\title{
A Study into the Key Issues in Preliminary Disciplinary Construction of Local Universities and Colleges
}

\author{
Huang Bin \\ School of Management \\ Wuhan Institute of Technology
}

\author{
Zhang Tong \\ School of Management \\ Jilin University
}

\author{
Liao Xiaoqian \\ School of Business \\ Lishui University
}

\begin{abstract}
This paper looks into the issues in the preliminary disciplinary construction of local universities and colleges, and discusses the thoughts and countermeasures in this regard from the aspects of team building, discipline management, quality of personnel training, construction of basic discipline and specialty cluster, and academic exchange, etc, and provides suggestions to deal with these issues.
\end{abstract}

Keywords- local universities and colleges; preliminary disciplinary construction; basic construction

\section{INTRODUCTION}

Disciplinary construction takes the lead in the construction of local universities and colleges. It is a fundamental and strategic system project of local universities and colleges embodying their improvement in teaching quality and academic level, a vital factor to measure their development potential and core competiveness, and a major symbol of their schooling level and characteristics, social popularity and overall strength. For disciplinary construction, it is necessary to follow the principles of disciplinary development, take Scientific Outlook on Development as guidance, emphasize basic disciplinary construction, take account of the demand of local social and economic development and adhere with the schooling and disciplinary development rules of local universities and colleges. However, in practice, some still have but hazy understandings on disciplinary construction, especially on what is disciplinary construction and how to implement and develop it in the early stage. Some even believe that disciplinary construction is nothing more than getting national topics and attracting high-end talents. This idea is wrong, for it simplifies a complex discipline project which needs systematization and effective supporting constructions into one simple process of $1+1=2$. This will surely lead to deviated meaning and value of disciplinary construction in terms of function setting, positioning, operation, service orientation and objects, etc, and consequently it will result into hidden danger and deeper problems, and eventually cause distorted disciplinary development. Therefore, basic disciplinary construction is the foundation of preliminary disciplinary construction, and it affects the objective and direction of future disciplinary development, so it is of vital research importance.

\section{STRESS THE PLANNING AND LAYOUT OF PRELIMINARY DISCIPLINARY CONSTRUCTION}

Most local universities and colleges possess distinctive characteristics due to their regional features. For this reason, these institutions must properly handle the relationships between preliminary disciplinary construction and specialty construction, preliminary disciplinary construction and scientific research, preliminary construction of key disciplines and academic degree construction, preliminary construction of basic disciplines and special disciplines, construction of discipline bases and personnel training. It is important to clarify research direction, actively nurture symbolic achievements, drive forward disciplinary construction by academic degree declaration, strengthen teacher building and academic exchange, and improve the security system for books and materials. Preliminary disciplinary construction is closely related with the survival and development of local universities and colleges. It is the lifeline of sustainable development and directly affects schooling interests, and it is school's fundamental and strategic task. Due to perception, foundation and capability, etc, many problems appear in the early stage of disciplinary construction. Therefore, as a local university with unique characteristics in undergraduate application, they should specify basic, advantageous and special disciplines, center on regional economic construction and social development, and actively explore a management model that suits the university positioning. Universities and colleges should rely on their advantages to survive and develop by their special disciplines, and gradually give play to their role in the regional social and economic development. Preliminary disciplinary construction is of vital importance to develop academic research, improve scientific and research levels and gain core competiveness. It should be known that preliminary disciplinary construction is a multi-aspect and tightly-structured system project.

\section{STRENGTHEN ASSOCIATION STUDY IN PRELIMINARY DISCIPLINARY CONSTRUCTION}

\section{A. Stress the construction of Echelon and talent mechanism}

Wrong understandings of team building should be corrected, such as the shallow idea that Echelon construction is only about bringing in talents, and that disciplinary construction is only about talents. On one hand, Echelon building is not only about bringing in talent; it also includes self-training which turns existing teachers into talents 
suitable for certain disciplines. Introduction and self-training are two irreplaceable aspects in Echelon building. On the other hand, without scientific management and appropriate use, introduced talents will become merely a decoration, a pile or isolated work, which will fail to reach the target of preliminary disciplinary construction. In addition, bad allocation of human resources also prevents the overall strength from working. Moreover, the living environment for talents is bad. There lacks humane care and understanding of the survival and development of talents, and insufficient understanding on talent innovation study and its principle. Talent is the incubator of innovations. The incubation needs space, period of time, and human, material and financial resources, and most of all it needs an atmosphere of talent culture. Without these consistencies in mind and action, it would only be a waste of talents. If disciplinary construction is a system project, then it is also a necessary system project of how to turn the attracted talents into an effective incubator. These above aspects show that the talent mechanism for local undergraduate university Echelon is of significance to preliminary construction and it is also important for the middle and late stages of disciplinary development.

Talent mechanism construction for discipline Echelon operation should be "people-oriented" and discipline team construction should base on framework building and security conditions. Echelon, innovation team or teaching team can get discipline members "stand in line", prevent them from scattering, clarify an individual's positioning, place and role in the discipline, and identify an individual's target direction, so as to realize overall enhancement of the discipline Echelon, and improve the strength and level of disciplinary structure. Scientific research team: disciplinary leader, disciplinary direction leader, and academic backbone staff and trainees. Leader should define three directions, and other members should determine their research interests accordingly. Personal positioning and objective: Doctorate or above, advanced professional title, domestic visiting scholar, international visiting scholar, disciplinary leader of provincial level or above. Teaching team: outstanding teacher at provincial level or above, tutor of student competition at provincial level or above, quality course director at provincial level or above, etc. The two teams and three directions are interrelated and interdependent to each other. Team building should realize good team development and satisfy the need of preliminary disciplinary construction. Management system should be built in accordance with principles. Preliminary disciplinary construction of local universities and colleges should reflect their development positioning, secure themselves with basic conditions, shoulder clear tasks and implement the specific construction contents.

Talent mechanism of discipline Echelon should aim at preliminary disciplinary construction and cultivation of highlevel talents, especially scientific research leaders and research teams. It focuses on providing talent and technical support for local economic, social and technical development, and strives to become an important innovative talent training base in the local innovation system, a knowledge innovation mainbody and an important force of scientific and technological innovation. In preliminary disciplinary construction, local universities and colleges are faced with multiple tasks, and its scientific research is featured with multi-layer teams, diversified orientations and extensive contents. However, due to most non-regional contents and impractical objects, the scientific research of local universities suffers from closed approach, scattered team, short-termed objective, and vague disciplinary ownership and positioning of members. A relatively stable discipline and direction are highly important to the optimization and functioning of the discipline team. For scientific research positioning, local universities and colleges should first consider the actual demand of local economic and social development, the utilization and development of historic and cultural heritage and resources, and stress disciplinary crossover and seepage and advantageous scientific research directions, so as to achieve a rational positioning. Scientific research leader is at the core of scientific research. They are of vital importance for innovations in knowledge, science and technology, and they constitute the decisive factors to promote scientific research and nurture characteristics. Specifically, scientific research leaders include discipline leader and academic leader. Lack of high-level and highcaliber scientific research leaders is a key factor that restricts the scientific research development of local universities and colleges, while lack of excellent talent utilization mechanism is the bottleneck of preliminary disciplinary construction. Therefore, Echelon disciplinary construction, especially the utilization of leaders should focus on developing key points, take supportive conditions as guidance, rely on vertical management, and take talent utilization mechanism as the primary mechanism of preliminary disciplinary construction, integrate scientific research teams and optimize team structure.

\section{B. Preliminary disciplinary construction and basic construction of overall scientific research level}

Preliminary disciplinary construction is the focus of local universities and colleges, and it aims to improve teaching quality and scientific research level, which are the symbol to measure a university's academic standing. Generally, local colleges are weak on this regard, for most of them are transformed from specialized colleges or are newly founded. They are in a pressing need for improvement in the overall academic level, which makes preliminary disciplinary construction all the more important.

First of all, academic research level needs comprehensive enhancement. Universities and colleges should strive for projects of ministerial or state levels; high-level papers (for domestic and international retrieval), monograph publication and government adoption should attach equal importance to quality and quantity. Second, academic exchange needs to be furthered. Universities and colleges should strengthen domestic and international academic exchanges and create more exchange channels. Academic exchange activities should be promoted. It should not only "invite in", but also "go out". Universities and colleges should ally with local institutions and enterprises as soon as possible, for the sake of initiating constructive governmental cooperation, as well 
as collaborations which serve preliminary disciplinary construction and satisfy social need. Members should be encouraged to participate in academic conferences at provincial level or above, deliver keynote speech, join academic organizations at provincial level or above and held key positions, so as to enlarge disciplinary influence and promote status. In the meanwhile, exchanges within the discipline should also be strengthened and comprehensively improved. Scientific research platform should be built. Produce-learn-research integration should be constructed under the ties of scientific research institutions. Disciplinary construction aims to meet the need of local social and economic development as well as technological innovation. Scientific research platform is the basis of scientific research, a fundamental condition for nurturing talents and an important method to obtain high-standard scientific research achievement. Therefore, it is important to realize rational resource allocation, overall cooperation and rolling development. Universities and colleges should fight for government support and investment, and build up research centers together with enterprises, so as to reinforce their own construction. For instance, they can build up research institutions, and hire honorary chairman from social organizations. They can solve practical problems based on their achievements to serve the discipline, profession and the society. They should also increase disciplinary propaganda to enhance influence and status. From the perspective of regional characteristics, local universities and colleges should learn to effectively carry out preliminary disciplinary construction, and explore the binding point between characteristic disciplines with regional features so as to create innovative disciplines.

\section{IMPROVE UNDERSTANDING ON CONSTRUCTION BASIS AND OBJECTIVES OF PRELIMINARY DISCIPLINARY CONSTRUCTION}

The foundation of preliminary disciplinary construction lies in the specialty cluster which supports the discipline. The objective of preliminary disciplinary construction is consistent with the ultimate object, which is to cultivate high-caliber talents who cater to the need of local economy and society. Maintaining such thinking can help sort out the guiding idea throughout disciplinary construction and better implement its purpose to serve local economic and social development. For this reason, two combinations must be realized to ensure implementation of preliminary disciplinary construction. The first is the combination of preliminary disciplinary construction and specialty construction (including course construction). Universities and colleges should improve their weak disciplinary support, and accelerate specialty construction of secondary discipline and the construction of relevant supportive specialty cluster, including updating existing specialties (curriculum) and applying new specialties. Disciplinary construction, specialty construction and course construction are three important aspects of local universities and colleges, and the standard, quality and status of these three aspects determine the schooling type, level and characteristics of an institution. Amongst specialty and course construction are the foundation of school's survival, and they are also the driving force and approach of preliminary disciplinary construction. The three aspects have their own focus, but they are also interrelated. Setting a new specialty affects the scale development of local universities and colleges, so it has to give consideration to social demand, moderate development and overall stability. Second is the combination of preliminary disciplinary construction and talent cultivation. In order to strengthen the relationship between characteristic preliminary disciplinary constructions with talent cultivation, it must ensure the basic guiding principle of Education Ministry that teachers of elementary specialty courses and specialty courses with senior or doctorate titles should be no less than $30 \%$, and that teachers with the same specialty background should take up a high percentage. In this way, it can guarantee that the teachers of elementary specialty courses and specialty courses have education background of first and second-level discipline, improve teaching quality, enhance postgraduate application rate, employment rate, and satisfaction of employer, so that eventually it can ensure the quality of the cultivated talents. Under the same discipline, students should make breakthroughs in scientific research innovation both in quality and quantity, and they should obtain landmark achievements. For instance, students should be assisted and guided to publish papers in project report or directly publish papers. Construction of laboratories and practice bases should be improved in quality and quantity, including adopting new lab application software, hiring industrial personnel as tutor, part-time teacher or lab teacher in laboratories and practice bases. Members of innovation team and teaching team should regularly carry out basic practices, investigations and researches in relevant enterprise departments.

Serving local economy construction is the foundation of school's survival. Local universities and colleges should notice the gap between the structure of professional and technical talent cultivation and the development requirements of current local industry, and quicken the steps in serving local construction. In a word, universities and colleges should narrow this gap and improve the effectiveness of serving local construction.

In a word, preliminary disciplinary construction is the foundational and strategic base of universities and research institutes [2], and also the core task of local universities and college. Preliminary disciplinary construction is a comprehensive, enduring and complex system project. Systematic research of the problems in preliminary disciplinary construction of local universities and colleges becomes the urgent demand of their self-development, talent cultivation, school competition and social service, and an indepth discussion of these problems can help work out the strategic thinking and countermeasures, so as to lay a solid foundation for leapfrog development.

\section{REFERENCES}

[1] Zhou Zude. Push forward All-round Development of University with Focus on Disciplinary Construction [J]. Higher Education in China, 2006, Vol.01 
[2] $\mathrm{Xu}$ Yachun. Practice and Exploration on Graduate Innovation Base Construction of Local Colleges and Universities [J]. Journal of Hunan Institute of Humanities, Science and Technology. 2012 (05)

[3] Han Xiangling, Jin Yisu, Peng Ying, Hu Xiaolong, Zhang Juhua, and Mu Kelang. Construct Open and Sharing Platform and Cultivate Topnotch Innovative Personnel [J]. China University Teaching. 2012 (05)

[4] Huang Zhengge. Ye Song. Problem Analysis in University Disciplinary Construction [J]. Higher Agricultural Education; 2004, Vol.09

[5] Yu Guoyang. Studies of the Discipline Development in Institutions of Higher Learning [J]. Journal of Guangzhou University (Social Science Edition) 2002, Vol.02

[6] Guo Hai. Patterns of Labor Market and Diversification of Higher Education [J]. Journal of Higher Education, 2005, Vol.04

[7] Tian Dingxiang, Hu Jianqiang. A Look into Some Problems in University Discipline Development [J], Hunan Social Sciences, 2003, Vol.02

[8] Ma Tao, Deng Pengtu. A Brief Analysis on the Meaning of Discipline Development in Institutions of Higher Learning [J]. Journal of Higher Education Research, 2004, Vol.01

[9] Yu Guoyang. Studies of the Discipline Development in Institutions of Higher Learning [J]. Journal of Guangzhou University (Social Science Edition) 2002, Vol.02

[10] Xie Guihua. Some Issues on Discipline Construction [J]. Journal of Higher Education. 2002, Vol.05

[11] Ma Jianhuai. Some Problems in Discipline Construction [J]. China Higher Education Research, 2001, Vol.07 